\title{
A Parallel Finite Volume Method for Aerodynamic Flows
}

\author{
Nigel Weatherill, Kaare Sørensen, Oubay Hassan, and Kenneth Morgan \\ Department of Civil Engineering, University of Wales, \\ Swansea SA2 8PP, Wales, U.K. \\ \{n.p.weatherill, cgsorens, o.hassan, k.morgan\} Oswansea.ac.uk \\ http://www. springer.de/comp/lncs/index.html
}

\begin{abstract}
The solution of 3D transient aerodynamic flows of practical interest is obtained by a finite volume approach, implemented on unstructured tetrahedral and unstructured hybrid meshes. The time discretised equation systems are solved by explicit iteration coupled with multigrid acceleration and the procedure is parallelised for improved computational performance. The examples presented involve an inviscid simulation of store release from a complete aircraft configuration and a viscous simulation of flow over an oscillating wing.
\end{abstract}

\section{Introduction}

In the aerospace industry, there are many practical applications that require the modelling of compressible time dependent flow with moving boundaries. These include the analysis of flutter, store release, buffeting and the deployment of control surfaces. In this paper, we present an approach for the simulation of such problems. Spatial discretisation of the governing flow equations is achieved by the adoption of an unstructured edge based finite volume method and this provides the geometric flexibility needed for flows involving complex geometries. The algorithmic implementation allows for the use of hybrid meshes of tetrahedra, prisms, pyramids and hexahedra. Hybrid meshes of this type are readily generated by using a modified unstructured tetrahedral mesh generator. For these transient problems, it is attractive to employ an implicit formulation, in which the time step used can be selected on accuracy criteria only. With implicit algorithms, it is important to adopt a formulation that avoids the requirement for working with large matrices and this is accomplished here by the use of a multigrid approach. The coarse meshes are nested and are constructed by agglomeration, with the control volumes merged together through an edge based method. Meshing difficulties encountered in the simulation of problems involving large displacement of the boundaries are overcome by employing a combination of mesh movement and local remeshing. The mesh is first moved, retaining the connectivities, as dictated by the geometry deflection. Any regions of bad mesh quality are then removed, creating holes which are remeshed using the unstructured mesh generator. The practical examples which are included show that the method is robust and applicable to geometries of a complication level experienced in industry. 


\section{Problem Formulation}

For modelling turbulent flows, the governing equations are taken to be the time dependent, Favre averaged [1], compressible Navier-Stokes equations. Here it is assumed that the time averaging suppresses the instantaneous fluctuations in the flow field caused by turbulence, while still being able to capture time dependency in the time scales of interest. The resulting equations can be expressed in integral form, on a three-dimensional Cartesian domain $\Omega(t)$, with closed surface $\partial \Omega(t)$, as

$$
\int_{\Omega(t)} \frac{\partial \boldsymbol{U}}{\partial t} \mathrm{~d} \boldsymbol{x}+\int_{\partial \Omega(t)} \boldsymbol{F}^{j} n_{j} \mathrm{~d} \boldsymbol{x}=\int_{\partial \Omega(t)} \boldsymbol{G}^{j} n_{j} \mathrm{~d} \boldsymbol{x}
$$

for $i, j=1,2,3$ and where the summation convention is employed. In this equation, $\boldsymbol{U}$ denotes the averaged vector of the conserved variables and $\boldsymbol{F}^{j}$ and $\boldsymbol{G}^{j}$ are the averaged inviscid and viscous flux components in direction $x_{j}$ respectively. The equation set is closed by assuming the fluid to be calorically perfect and the turbulent viscosity is determined by the Spalart-Allmaras model [2].

\section{Discretization Procedure}

Hybrid meshes are generated using a merging procedure, constructing complex elements from an initial simplex mesh. Quadrilateral elements are generated on the wall surfaces. This is accomplished by an indirect method of combining triangles, coupled with a split scheme to guarantee meshes consisting of quadrilateral elements only. The advancing layer method [3] is then employed to generate stretched elements adjacent to those boundary surface components which represent solid walls. The height and number of layers is specified by the user in such a way that the expected boundary layer profile should be capable of being adequately represented. The implementation starts from a triangular mesh on the surface of the wall, which means that each quadrilateral element has to be treated as two triangles in the generation process. The layers are constructed by generating points along prescribed lines and connecting the generated points, by using advancing front mesh generation concepts, to form tetrahedral elements. Point generation ceases before the prescribed number of layers is reached if an intersection appears or if the local mesh size is close to that specified in the userspecified mesh distribution function. In these layers, tetrahedra are merged to form prisms, pyramids and hexahedra. The remainder of the domain is meshed using a standard isotropic Delaunay procedure [4]. Tetrahedra are merged to form prisms, pyramids and hexahedra. On the resulting mesh, the governing equations are discretised by using a finite-volume method, in which the unknowns are located at the vertices of the mesh and the numerical integration is performed over dual mesh interfaces [5]. For problems in which the control volumes may change with time, it is convenient to apply the general Leibnitz rule to the time derivative term in equation (1). In this case, the governing equations 
may be expressed as

$$
\frac{\mathrm{d}}{\mathrm{d} t} \int_{\Omega(t)} \boldsymbol{U} \mathrm{d} \boldsymbol{x}+\int_{\partial \Omega(t)}\left(\boldsymbol{F}^{j}-v_{j} \boldsymbol{U}\right) n_{j} \mathrm{~d} \boldsymbol{x}=\int_{\partial \Omega(t)} \boldsymbol{G}^{j} n_{j} \mathrm{~d} \boldsymbol{x}
$$

where $\boldsymbol{v}=\left(v_{1}, v_{2}, v_{3}\right)$ is the velocity of the control volume boundary.

\subsection{Spatial Discretization}

A dual mesh is constructed by connecting edge midpoints, element centroids and face-centroids in such a way that only one node is contained within each control volume [6]. Each edge of the grid is associated with a surface segment of the dual mesh interface between the nodes connected to the edge. This surface is defined using triangular facets, where each facet is connected to the midpoint of the edge, a neighbouring element centroid and the centroid of an element face connected to the edge. With this dual mesh definition, the control volume can be thought of as constructed by a set of tetrahedra with base on the dual mesh. To enable the integration over the surface of the control volumes, a set of coefficients is calculated for each edge using the dual mesh segment associated with the edge. The discrete equations are then formed by looping over the edges in the mesh and sending edge contributions to the nodes. The task of finding edge coefficients that ensure that the resulting scheme is geometrically conservative is not trivial. Here, geometric conservation is assured by modifying the approach of Nkonga and Guillard [7] to handle the case of dual meshes constructed of assemblies of triangular facets. Finally, as the basic procedure is essential central difference in character, a stabilizing dissipation must be added and this is achieved by a method of JST type [8].

\subsection{Mesh Movement}

For the simulation of a problem involving moving boundaries, the mesh must deform to take account of the movement. This is usually achieved by fixing the mesh on the far field boundary, while moving the mesh nodes on the geometry in accordance with the movement. The interior mesh nodes are moved accordingly to achieve the desired mesh quality. A number of different mesh movement approaches have been investigated in the literature but the approach used here assumes that the edges of the mesh behave like springs connecting the nodes [9]. The nodes on the moving geometry are moved in small increments, with the interior nodes being moved to ensure internal equilibrium for each increment. Typically 50 increments are employed with this procedure. This approach is robust and fast, usually requiring about $10-15 \%$ of the total CPU-time required to solve the system time-accurately. With this approach, it is also possible to obtain a certain level of control over the mesh movement, by varying the spring constants of the edges. 


\subsection{Local Remeshing}

Often, it is impossible to avoid remeshing if the necessary mesh quality is to be maintained. The regions in which mesh movement is inappropriate are, usually, relatively small, however, and this is utilised by applying local remeshing only. The regions that need remeshing are found by using a mesh quality indicator which is taken to be the ratio of the volume of an element at the current time level and the element volume in the original mesh. According to the values produced by this indicator, certain elements are removed, creating one or several holes in the mesh. Each of these holes is meshed in the normal manner by using a Delaunay scheme, with the triangulation of the hole surface being taken as the surface triangulation [9]. In certain circumstances, it may not be possible to recover the hole surface triangulation and, in this case, another layer of elements is removed from the original mesh and the process is repeated. The unknown field is transferred from the previous mesh level by linear interpolation.

\subsection{Time Discretisation}

The spatially discretised equation at node $I$ on the mesh is of the form

$$
\frac{\mathrm{d}}{\mathrm{d} t}\left(V_{I} \boldsymbol{U}_{I}\right)=\boldsymbol{R}_{I}
$$

where $V_{I}$ is the control volume associated with node $I$ and $\boldsymbol{R}_{I}$ denotes the assembled edge contributions to this node. A three level second order accurate time discretisation is employed and this equation is represented in the form

$$
\frac{3}{2}\left[V_{I} \boldsymbol{U}_{I}\right]^{n+1}-2\left[V_{I} \boldsymbol{U}_{I}\right]^{n}+\frac{1}{2}\left[V_{I} \boldsymbol{U}_{I}\right]^{n-1}=\Delta t \boldsymbol{R}_{I}^{n}
$$

where the superscript $n$ denotes and evaluation at time $t=t_{n}$ and $t_{n}=t_{n-1}+\Delta t$, $t_{n-1}=t_{n-2}+\Delta t$. A first order backward time discretisation scheme is used for simulations which require the use of remeshing.

\section{Solution Procedure}

\subsection{Multigrid Scheme}

At every time step, the discretisation procedure results in a numerical solution of an equation system and this solution is obtained by the FAS multigrid scheme [10]. The approach avoids the requirement for linearisation of the discrete system and eliminates the need to store a Jacobian matrix. Assuming nested meshes, the restriction operator that maps the coarse mesh quantities to the fine mesh is constructed as a linear restriction mapping. This implies that the values on the coarse mesh are produced as the weighted average of the fine mesh values. For prolongation, a simple point injection scheme is adopted. The relaxation scheme that is employed in conjunction with the multigrid procedure is a three-stage Runge-Kutta approach with local time stepping, and coefficients $0.6,0.6$ and 1.0 . 


\subsection{Coarse Mesh Generation}

The assumption of nested coarser meshes is met by generating the coarser meshes by agglomeration. The agglomeration approach works on the dual mesh, merging control volumes to achieve the local coarseness ratio required $[11,12]$. This approach is purely edge-based and can therefore be applied on any type of mesh after the edge-based data structure has been assembled. Since no mesh generation issues are raised in the procedure, the scheme is completely stable. It is also fast and requires little memory overhead.

\subsection{Implementation}

The computational implementation consists of four main components: (i) mesh movement: a geometry file and the surface movement information is prescribed; the internal nodes of the mesh are positioned employing the mesh movement algorithm; (ii) remeshing: the local quality of the moved mesh is investigated and remeshing is performed if required; (iii) preprocessing: the edge-based data structure is set up from the mesh information, the coarse grids are generated and the inter-grid interpolation arrays for the multigrid procedure are constructed; (iv) equation solving: the output from the preprocessor is accessed and the time dependent discrete equations are solved for one physical time step. These components are loosely coupled, employing a driving script and file communication. Such a loose coupling will, inevitably, adversely affect the solution time compared to implementations which do not require I/O communication. However, since relatively few time steps are required for the implicit schemes, this effect is usually small.

\subsection{Parallelisation}

Parallelisation of the solution procedure is necessary to make best use of multiprocessor computer platforms. For parallelisation, the mesh is created sequentially and then split into the desired number of computational domains using the METIS library [13]. This procedure essentially colours the nodes in the mesh according to the domain to which they belong. A preprocessor renumbers the nodes in the domains and generates the communication data required. The edges of the global mesh are placed in the domain of the nodes attached to the edge, or if the nodes belong to different domains, as occurs with an inter-domain edge, the edge is placed in the domain with the smallest domain number. The edges are renumbered in each domain, placing the inter-domain edges first in the list. On the inter-domain boundaries, the nodes are duplicated so that each domain stores a copy of the nodes appearing in the edge list. These ghost nodes are allocated a place in memory to store the nodal contributions from adjacent domains. If the node belongs to the domain, it is termed a real node. The communication data structure consists of two registers for each domain combination on each grid level. These registers are termed the ghost node register and real node register and simply store the local node indexes of the ghost and real nodes respectively. 
The data structure of the boundary edges is created in an analogous fashion. The solution procedure consists, essentially, of loops over edges and loops over nodes and these are readily parallelised. A global parallelisation approach has been followed, in which agglomeration is allowed across domain boundaries. While requiring communication to be performed in the intergrid mappings, this approach yields an implementation equivalent to a sequential code. The approach does not guarantee balanced coarse meshes, but this is of secondary importance as the coarse meshes are significantly smaller than the finest mesh.

\section{$5 \quad$ Examples}

Two examples are presented to illustrate the capability of the proposed procedure. The first example is an inviscid simulation involving a complete aircraft configuration while the second example is the simulation of turbulent flow over an wing.

\subsection{Store Release}

The first example is the simulation of the release of two stores from an aircraft configuration. The stores are assumed to be completely filled with a substance of density 1250 times that of the free stream air. The free stream Mach number is 0.5 , the angle of attack is zero and the Froude number is 1333 . The rigid body movement of the stores is calculated by integrating the pressure field over the stores and using a second order accurate time integration. Local remeshing is employed and each mesh consists of about 2.7 million tetrahedral elements. The resulting surface pressure distribution, at four equally spaced time intervals, is shown in Figure 1.

\subsection{Oscillating Wing}

The results of a viscous flow simulation over an oscillating ONERA M6 wing are now considered. The free stream Mach number is 0.84 and the initial angle of attack is 3.06 degrees. The Reynolds number, based on the mean geometric chord, is set to $1.2 \times 10^{7}$ and turbulence is triggered at $5 \%$ chord. A hybrid mesh, consisting of 1641721 prisms, 26139 pyramids and 1826751 tetrahedra is employed. The number of edges in this mesh is 4961611 . The wing root is held fixed, while the wing tip is subjected to a prescribed sinusoidal movement with 3 degrees amplitude. Between these two sections, the amplitude varies linearly along the wing span and the axis of rotation is along the quarter chord line of the wing. The Strouhal number for this movement is 3.10 and 16 time steps were performed for each cycle. At each time step, 150 multigrid cycles were used. One full movement cycle, employing 16 R14000 cpus for the solver module and one R14000 cpu for the mesh movement and preprocessor modules, requires about 12 hours of wall clock time. A plot of the resulting lift polar is shown in Figure 2. 


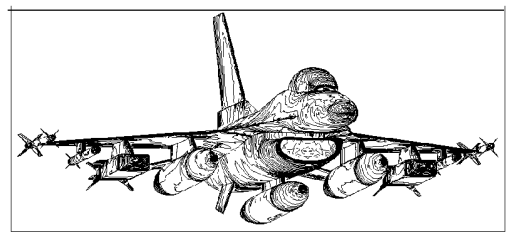

(i)

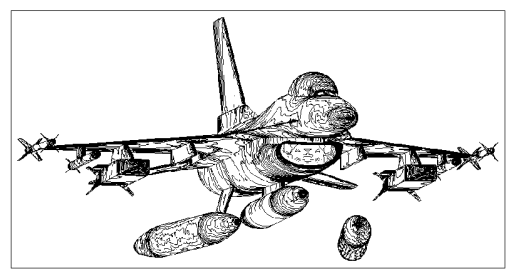

(iii)

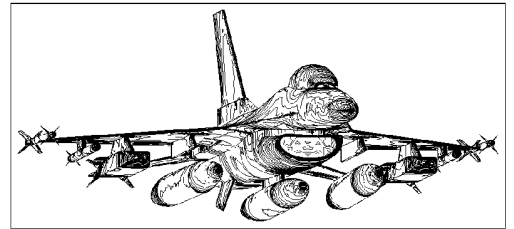

(ii)

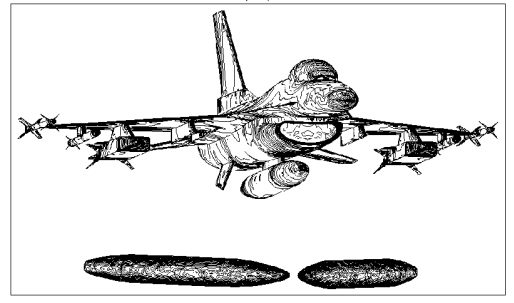

(iv)

Fig. 1. Surface pressure distribution, at 0.29 second intervals, following store release from an aircraft

\section{Conclusion}

A method for solving compressible aerodynamic flow problems involving moving geometries has been described. A parallelised multigrid implicit time stepping approach is adopted which, when coupled with a mesh-movement/local remeshing capability, provides an accurate and robust technique for solving practical aerodynamic problems of this type.

\section{Acknowledgements}

Kaare Sørensen acknowledges the sponsorship of The Research Council of Norway, project number $125676 / 410$.

\section{References}

1. A. Favre, Equation des gaz turbulents compressibles, Journal de Mechanique, 4, 361-390, 1965

2. P. R. Spalart and S. R. Allmaras, A one equation turbulent model for aerodynamic flows, AIAA Paper 92-0439, 1992

3. O. Hassan, K. Morgan, E. J. Probert and J. Peraire, Unstructured tetrahedral mesh generation for three-dimensional viscous flows, International Journal for Numerical Methods in Engineering, 39, 549-567, 1996 


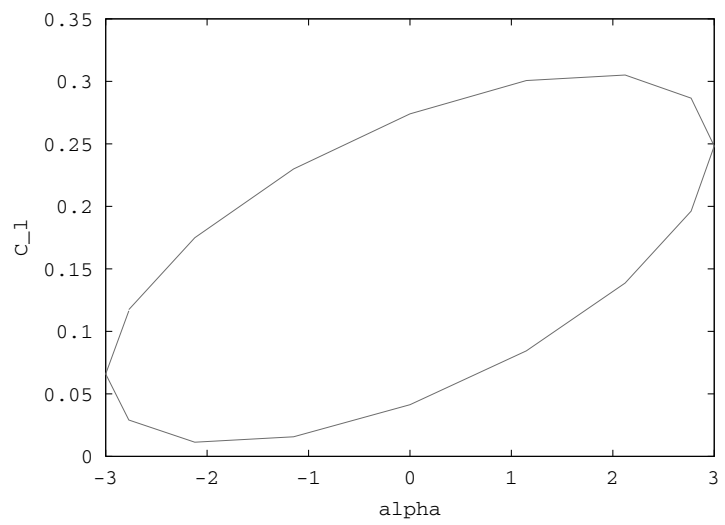

Fig. 2. The lift polar for turbulent flow over an oscillating ONERA M6 wing

4. N. P. Weatherill and O. Hassan, Efficient three-dimensional Delaunay triangulation with automatic boundary point creation and imposed boundary constraints, International Journal for Numerical Methods in Engineering, 37, 2005-2039, 1994

5. K. A. Sørensen, A multigrid accelerated procedure for the solution of compressible fluid flows on unstructured hybrid meshes, $P h D$ Thesis, C/Ph/251/01, University of Wales Swansea, 2002

6. M. Vahdati, K. Morgan and J. Peraire, Computation of viscous compressible flows using an upwind algorithm and unstructured meshes, in S. N. Atluri, editor, Computational Nonlinear Mechanics in Aerospace Engineering, AIAA, Washington, 479-505, 1992

7. B. Nkonga and H. Guillard, Godunov type method on non-structured meshes for three-dimensional moving boundary problems, Computational Methods in Applied Mechanical Engineering, 113, 183-204, 1994

8. A. Jameson, W. Schmidt and E. Turkel, Numerical simulation of the Euler equations by finite volume methods using Runge-Kutta timestepping schemes AIAA paper 81-1259, 1981

9. O. Hassan, E. J. Probert, K. Morgan and N. P. Weatherill, Unsteady flow simulation using unstructured meshes, Computer Methods for Applied Mechanical Engineering, 189, 1247-1275, 2000

10. A. Brandt, Multi-level adaptive solutions to boundary value problems Mathematics of Computation, 21, 333-390, 1977

11. M. H. Lallemand, H. Steve and A. Dervieux, Unstructured multigridding by volume agglomeration: Current status, Computers \& Fluids, 21, 397-433, 1992

12. D. J. Mavriplis and V. Venkatakrishnan, A 3D agglomeration multigrid solver for the Reynolds-averaged Navier-Stokes equations on unstructured meshes, International Journal for Numerical Methods in Fluids, 23, 527-544, 1996

13. G. Karypis and V. Kumar, Multilevel k-way partitioning scheme for irregular grids, Journal of Parallel and Distributed Computing, 48, 96-129, 1998 